therapies acted as might be expected for instance, nutlin-3a, which inhibits an interaction between wild-type p53 protein (which is encoded by TP53) and a binding partner, effectively killed cells in organoids in which TP53 was not mutated. By contrast, sensitivity to general chemotherapies did not correlate well with mutational status. The heterogeneity of drug response among samples from different regions of the same tumour provides a particularly sobering demonstration of the challenges of therapeutic resistance in cancer.

Overall, Roerink and colleagues' study is a remarkable demonstration of the ability of organoid technologies to amplify rare cancer-cell populations and enable deep analyses. However, the current approaches have limitations. For instance, organoids do not provide a wholly accurate snapshot of the situation in a tumour, because the growth of organoids involves cell proliferation, which itself can lead to further mutations. To get around this problem, Roerink et al. focused on mutations present in all cells of an organoid in their analysis. Another caveat is that organoid culture methods might also select for cells particularly adapted for in vitro growth, potentially correlating with the most proliferative or aggressive cells.

Nevertheless, this and other organoid methods should in the future prove invaluable tools for the genetic and functional dissection of cancer. A big help in this endeavour will be biobanks of patient-derived organoids ${ }^{9,10,13}$, which have been established in the past few years to encapsulate the genetic diversity of cancers from different anatomical sites. Eventually, organoids might also incorporate components of the tumour microenvironment, enabling more-holistic cancer modelling. Finally, Roerink and colleagues' method could be generalized beyond cancer to enable the expansion of limiting amounts of biopsy tissue from inflammatory, metabolic or genetic disorders. Here, as in cancer, this approach could reveal cellular heterogeneity and inform treatment strategies.
Calvin J. Kuo is in the Division of Hematology, Stanford University School of Medicine, Stanford Cancer Institute, Stanford, California 94305, USA. Christina Curtis is in the Division of Oncology and the Department of Genetics, Stanford University School of Medicine, Stanford, California 94305, USA. e-mails: cjkuo@stanford.edu; cncurtis@stanford.edu

1. Roerink, S. F. et al. Nature 556, 457-462 (2018).

2. Sottoriva, A. et al. Nature Genet. 47, 209-216 (2015)

3. Sun, R. et al. Nature Genet. 49, 1015-1024 (2017).

4. Vogelstein, B. et al. Science 339, 1546-1558 (2013).

5. Nik-Zainal, S. et al. Cell 149, 994-1007 (2012).

6. Bivona, T. G. \& Doebele, R. C. Nature Med. 22 , 472-478 (2016).

7. Sharma, P., Hu-Lieskovan, S., Wargo, J. A. \& Ribas, A. Cell 168, 707-723 (2017).

8. Gao, D. et al. Cell 159, 176-187 (2014).

9. van de Wetering, M. et al. Cell 161, 933-945 (2015)

10.Boj, S. F. et al. Cell 160, 324-338 (2015).

11.Fujii, M. et al. Cell Stem Cell 18, 827-838 (2016).

12.Sachs, N. et al. Cell 172, 373-386 (2018).

13.Sato, T. et al. Gastroenterology 141, 1762-1772 (2011).

This article was published online on 11 April 2018.

\title{
Transition states that allow cancer to spread
}

\section{Cancers of epithelial-cell origin often contain some tumour cells that have acquired traits of mesenchymal cells. How this leads to cancer spread has now been illuminated in mouse models. SEE ARTICLE P.463}

\section{ERIK W. THOMPSON}

\section{\& SHIVASHANKAR H. NAGARAJ}

$\mathrm{I}$ $\mathrm{n}$ cancers that arise from epithelial cells, some of the tumour cells adopt features of mesenchymal cells and lose their epithelial characteristics. This phenomenon is known as the epithelial-to-mesenchymal transition (EMT), and the emergence of cells with mesenchymal features is often associated with a poorer prognosis for patients. How this transition occurs and the implications of EMT for metastasis, the process by which cancer spreads, are not fully understood. On page 463 , Pastushenko et al. ${ }^{1}$ report an analysis of mouse tumours undergoing EMT, revealing that distinct cell populations can be identified during this transition.

Cancers of epithelial tissues are called carcinomas and can give rise to cells with mesenchymal properties, such as an elongated shape. These mesenchymal cells have been implicated $^{2}$ in tumour metastasis. The EMT process is also associated with cancer stem cells found in breast cancer and other cancer types, and is linked to the presence of the circulating tumour cells in the bloodstream that are a hallmark of tumour-relapse risk ${ }^{3}$. In numerous mouse models ${ }^{2}$, prevention of EMT or its reversal - a process known as MET, in which mesenchymal cells transition back to epithelial cells - can reduce the stem-cell-like properties of tumour cells and thereby reduce their ability to metastasize.

Although some studies ${ }^{4,5}$ indicate that not all tumour metastases require EMT, considerable amounts of data implicate ${ }^{6}$ the process of MET as a requirement for metastatic cells to successfully colonize locations beyond the initial tumour site. One way to address this controversy would be to gain a better understanding of how the EMT process occurs.

A hybrid EMT state, also known as a metastable state or partial EMT, in which individual cells express both epithelial and mesenchymal markers, has often been observed in carcinoma ${ }^{7}$, developmental processes and wound healing ${ }^{8}$. One model for how EMT might occur is that cells make a gradual transition from one state to the other along a continuous spectrum of change in which cells lose epithelial characteristics and concurrently gain mesenchymal ones. This is consistent with gene-expression analysis of cell lines from various types of carcinoma ${ }^{9}$. However, computational-modelling studies ${ }^{7}$ and in vitro observations ${ }^{8}$ indicate instead that distinctive, stable, long-lasting cell populations could represent discrete intermediate stages of EMT.

Pastushenko and colleagues investigated whether stable EMT states in tumours could be identified in vivo. The authors used genetically engineered mice that provide a model system in which to study skin carcinoma and have tumour cells ${ }^{10}$ that are known to exhibit EMT. Pastushenko et al. studied cells that had lost expression of the epithelial-cell marker EpCAM, and analysed the expression of more than 170 cell-surface proteins to try to identify useful markers of cell subpopulations. They then focused on three of these markers (CD61, CD51 and CD106) that are characteristic of a mesenchymal-cell state. This enabled the authors to identify distinct cell populations that had lost expression of the epithelial-cell marker EpCAM and expressed different combinations of these three mesenchymal markers.

Using the three markers as a tool to enable the isolation of cell populations present when EMT occurs, the authors isolated and characterized six distinctive cell subpopulations that lacked EpCAM expression. The authors used an impressive array of biomolecular analyses to assess the cellular characteristics of these populations, including their metastatic traits and their state of cellular differentiation. This work provides in vivo evidence of stable cell populations representing intermediate EMT states.

The authors also identified comparable cell subpopulations, characterized by the same markers, in both a mouse mammarytumour model and human tumour samples transplanted into mice, suggesting that these subpopulations represent stable transitional cellular states found in different cancer types. 


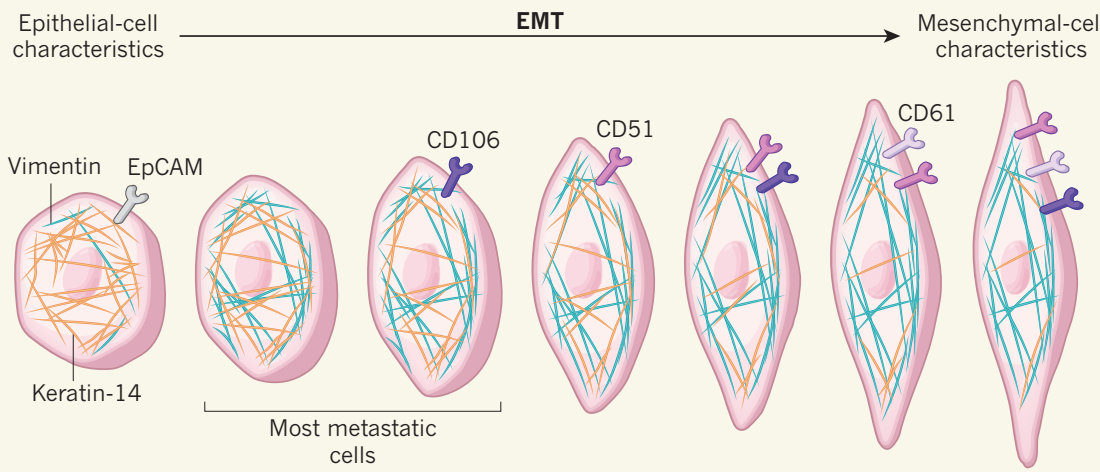

Figure 1 | Populations of cancer cells in transition states. Cancers that arise from epithelial cells often contain some tumour cells that have acquired mesenchymal-cell characteristics, such as an elongated shape and the expression of genes associated with mesenchymal cells. This change is termed the epithelial-to-mesenchymal transition (EMT). Whether this transition occurs as a gradual, continuous change or by discrete stages is debated. Pastushenko et al. ${ }^{1}$ report mouse studies consistent with the latter, in which they identified cell populations in vivo that represent stable, distinct intermediates in this transition. The authors analysed cells that do not express the epithelial-cell receptor protein EpCAM, and isolated cell populations on the basis of their expression of the mesenchymal-cell receptor proteins CD51, CD61 and CD106. They monitored the level of proteins characteristic of the epithelial-cell state (such as keratin-14, yellow) or of the mesenchymal-cell state (such as vimentin, blue), and found cells that exhibited a hybrid of epithelial and mesenchymal characteristics. Cells that expressed EpCAM had low levels of vimentin and high levels of keratin-14. Once EpCAM expression was lost, the level of vimentin increased sharply and the level of keratin-14 declined incrementally as cell populations became more mesenchymal in character. The authors identified two cell populations that were the most likely to spread and form tumours elsewhere in the process called metastasis.

This finding might have clinical relevance. The authors' work therefore provides a platform for future investigations of this phenomenon in other cancer models, including studies of the regulatory networks that control these states. Such investigations might improve understanding of the molecular and cellular features that underlie the tumour-cell capabilities associated with these states.

Do these subpopulations exhibit behaviours that might offer therapeutic opportunities? One of the authors' most striking findings, made in the mouse skin-carcinoma and mammary-tumour models, is that when comparing these cell subpopulations, two of them stood out as having a substantially higher metastatic potential (Fig. 1). This raises the question of whether these highly metastatic cells could be specifically targeted with drugs to arrest tumour progression. Interestingly, none of the stable subpopulations was superior to the others in terms of tumour-initiating capability or proliferation rate. Also, each of the subpopulations that had acquired mesenchymal characteristics exceeded the metastatic capabilities of the epithelial-cell population. These findings are relevant to current debates in this field, which include the question of whether a cycle of EMT followed by MET is required for successful metastatic colonization of a secondary location $^{6,11}$.

The authors' work reveals the progressive acquisition of EMT features in EMT hybrid cells, supporting the previously proposed ${ }^{8}$ idea of stable transitional states. A process of stepwise change is supported by Pastushenko and colleagues' data, indicating that distinct transcriptional and signalling processes govern intermediate aspects of EMT. The different cell subpopulations had characteristic transcriptional signatures regulated by distinct transcription-factor proteins, underscoring the reproducible and meticulously regulated nature of these hybrid cells.

Although these hybrid cell populations are a stable presence in tumours, the authors found that these cells also maintain a high degree of plasticity, given their ability to undergo MET and revert back to an epithelial-cell state. However, the authors found that the cell subpopulations that were best at undergoing MET during tumour metastasis to the lung in mice were not the most metastatic populations, which will fuel the debate ${ }^{6}$ about whether MET is a requirement for metastasis.

Elucidation of the circuitry and signalling feedback loops that might stabilize ${ }^{7,12}$ these distinct cellular states will be a worthy goal for future work. Studies of such cellular intermediates in patients' tumours, circulating tumour cells and metastases would be an ideal way to extend this work in a clinical context. If EMT states can be characterized for a wide range of human tumours, this might offer a way to enhance personalized approaches for cancer treatment. The development of specific technologies to identify human EMT states might allow clinicians to predict a tumour's metastatic potential and thereby plan the most effective treatment regimen.

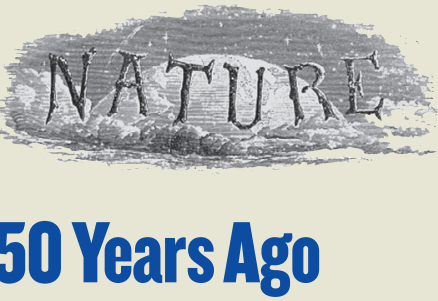

Can experiments with bacteria or strips of gut help to explain the behaviour of the drug addict? Are drugs sought because of their special pharmacological properties, or as a form of social currency? ... Such were the questions tackled at a symposium on the scientific basis of drug dependence ... Sir Aubrey Lewis reviewed past attitudes and terminology. Although "physical" dependence could be reliably defined, "psychological" dependence was a dangerously woolly concept. Thus psychogenic polydipsia might be cited to prove water a drug of dependence. He underlined the need for a sense of proportion by quoting the scathing denunciation, by two authorities a generation ago, of the danger to mankind of that menacing beverage, tea.

From Nature 27 April 1968

\section{Years Ago}

A disease known as "trench fever" has been very frequent among the troops on the Western front. It is characterised by recurrent attacks of fever of short duration ... and followed generally by acute pain in the shins and frequently by dilatation and disordered action of the heart. A committee ... was instituted to investigate the causation and spread of the disease ... various circumstances implicated the louse, and experiments were made on this hypothesis. Lice were allowed to feed on patients in all stages of the disease, and were then allowed to bite healthy volunteers; the result was negative. Next the excreta of lice similarly infected were applied to a scarified area of skin, and in from six to ten days after, all the five volunteers ... developed trench fever. From these experiments it is evident that the bite alone of the louse does not produce trench fever, but that when the excreta of infected lice are scratched into the skin the disease is produced. From Nature 25 April 1918 
Erik W. Thompson and

Shivashankar H. Nagaraj are in the Institute of Health and Biomedical Innovation, and the School of Biomedical Sciences, Queensland University of Technology, Brisbane QLD 4059, Australia. They are also at the Translational Research Institute, Woolloongabba, Australia. e-mails:e2.thompson@qut.edu.au; shiv.nagaraj@qut.edu.au

1. Pastushenko, I. et al. Nature 556, 463-468 (2018)

2. Chaffer, C. L., San Juan, B. P., Lim, E. \&

Weinberg, R. A. Cancer Metastasis Rev. 35, 645-654 (2016).

3. Francart, M. E. et al. Dev. Dyn. 247, 432-450 (2017).
4. Zheng, X. et al. Nature 527, 525-530 (2015).

5. Fischer, K. R. et al. Nature 527, 472-476 (2015).

6. Brabletz, T., Kalluri, R., Nieto, M. A. \& Weinberg, R. A Nature Rev. Cancer 18, 128-134 (2018).

7. Jolly, M. K. et al. Oncotarget 7, 27067-27084 (2016).

8. Nieto, M. A., Huang, R. Y.-J., Jackson, R. A. \& Thiery, J. P. Cell 166, 21-45 (2016).

9. Tan, T. Z. et al. EMBO Mol. Med. 6, 1279-1293 (2014).

10.Latil, M. et al. Cell Stem Cell 20, 191-204 (2017)

11.van Denderen, B. J. W. \& Thompson, E. W. Nature 493, 487-488 (2013)

12.Bracken, C. P. et al. Cancer Res. 68, 7846-7854 (2008).

This article was published online on 18 April 2018

\section{QUANTUM PHYSICS}

\section{Entangled vibrations in mechanical oscillators}

Two experiments have demonstrated entanglement - non-classical correlations between remote mechanical systems comprising billions of atoms. The results could advance our understanding of quantum physics. SEE LETTERS P.473 \& P.478

\section{ANDREW ARMOUR}

I $\mathrm{n}$ the quantum world, the properties of particles can be correlated in an extremely strange way. Measurements on one particle can influence the properties of another, even if the two particles are far apart. Such behaviour is known as entanglement and was initially so paradoxical that many people, including Albert Einstein ${ }^{1}$, thought that the underlying theory must be incomplete. However, experiments have verified the counter-intuitive properties of entanglement ${ }^{2}$, and physicists have got used to the idea, recognizing that it could be exploited to develop innovative forms of technology. On pages 473 and 478 , respectively,
Riedinger $e t a l{ }^{3}$ and Ockeloen-Korppi et $a l^{4}{ }^{4}$ take the exploration of entanglement in a new direction. They entangle the vibrations of a pair of remote mechanical oscillators, each of which contains billions of atoms.

Mechanical oscillators, such as a mass on a spring or the head of a drum, are familiar objects. They respond to being pushed out of equilibrium by vibrating back and forth at a fixed frequency. Because motion can be generated in lots of different ways - using light, electrical currents or even gravity mechanical oscillators are highly versatile. Consequently, they have many applications, for example in the detection of weak forces ${ }^{5}$.

Uncovering signatures of quantum a

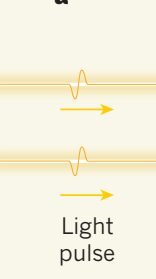

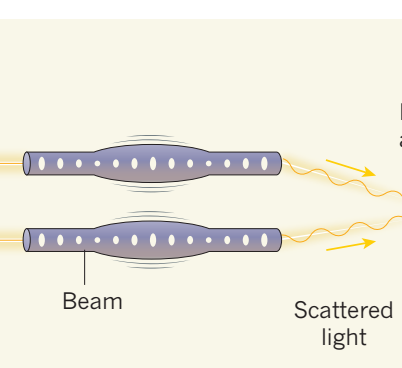

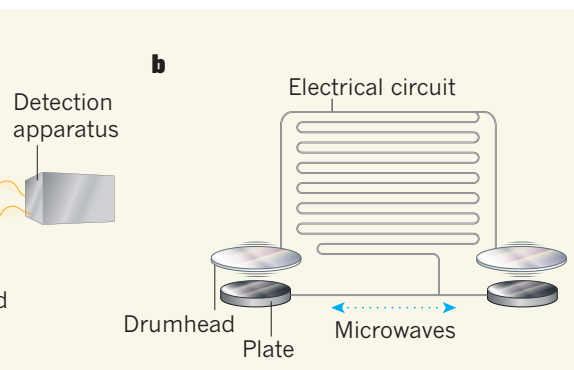

Figure 1 | Entanglement of two types of mechanical oscillator. Riedinger et al. ${ }^{3}$ and Ockeloen-Korppi et al. ${ }^{4}$ report entanglement (non-classical correlations) between the vibrations of two remote micrometre-scale mechanical oscillators. a, Riedinger and colleagues used oscillators in the form of silicon beams. Each beam contained small holes designed to trap light, that coupled to rapid oscillations in the beam's width. The authors achieved entanglement by shining pulses of light on the beams and detecting the light that was scattered. b, By contrast, Ockeloen-Korppi and colleagues used metal drumheads that vibrated up and down above fixed metal plates. The drumheads and the plates were connected by an electrical circuit. The authors injected microwaves into the circuit; these bounced back and forth between the oscillators, coupling the drumheads and giving rise to entanglement. 